\title{
Cardiopulmonary Resuscitation, Chest Compression Only and Teamwork From the Perspective of Medical Doctors, Surgeons and Anesthesiologists
}

\author{
Irena Krajina ${ }^{1}$; Slavica Kvolik ${ }^{1,2, *}$; Robert Steiner ${ }^{1,3}$; Kristina Kovacevic ${ }^{1}$ Ivan Lovric ${ }^{1,4}$ \\ ${ }^{1}$ Faculty of Medicine, University of Osijek, Osijek, Croatia \\ ${ }^{2}$ Department of Anesthesiology and ICU, Osijek University Hospital, Osijek, Croatia \\ 3 Department of Cardiology, Osijek University Hospital, Osijek, Croatia \\ ${ }^{4}$ Department of Surgery, Osijek University Hospital, Osijek, Croatia \\ ${ }^{*}$ Corresponding Author: Slavica Kvolik, Department of Anesthesiology and ICU, Faculty of Medicine, University of Osijek, J. Huttlera 4, 31000 Osijek, Osijek, Croatia. Tel/Fax: +38- \\ 531206444, E-mail:skvolik@mefos.hr
}

Received: February 14, 2014; Revised: November 15, 2014; Accepted: December 13, 2014

\begin{abstract}
Background: New resuscitation guidelines that were proposed by the European Resuscitation Council in 2010 have introduced a new method of cardiopulmonary resuscitation (CPR) by chest compressions only for untrained individuals.

Objectives: We conducted this study to evaluate differences in attitudes towards CPR among medical doctors, surgeons and anesthesiologists in Osijek University Hospital. A call for help, chest-compression-only resuscitation, mouth-to-mouth ventilation and team-work were recognized as critical points that may influence the outcome. Unfamiliarity with these methods may be indicative of a lack of education in resuscitation and may result in poor outcomes for victims.

Patients and Methods: An anonymous survey was conducted on 190 medical professionals: 93 medical doctors, 70 surgeons, and 27 anesthesiologists during year 2012 (mean age 41.9 years). The questions were related to previous education in resuscitation, current resuscitation practices and attitudes towards cardiopulmonary resuscitation. Data were analyzed using ANOVA and Fisher exact test. A P value of $<0.05$ was considered statistically significant.

Results: The only difference between groups was regarding the male and female ratio, with more male surgeons ( 45,55 , and $11, \mathrm{P}<0.001$ ). All doctors considered CPR as important, but only anesthesiologists knew how often guidelines in CPR change. Approximately $45 \%$ of medical doctors, $48 \%$ of surgeons and $77 \%$ of anesthesiologists reported that they have renewed their knowledge in CPR within the last five years, whereas 34\%, 25\% and 22\% had never renewed their knowledge in the CPR $(P=0.01$ between surgeons anesthesiologists). Furthermore, chest-compression-only was recognized as a valuable CPR technique by $25.8 \%$ of medical doctors, $14.3 \%$ of surgeons and $59.3 \%$ of anesthesiologists $(\mathrm{P}<0.001)$. Anesthesiologists estimated a high risk of infection transmission (62\%) and were more likely to refuse mouth-to-mouth ventilation when compared to surgeons $(25 \%$ vs.10\%, $\mathrm{P}=0.01$ ). Anesthesiologists are most often called for help by their colleagues, only rarely surgeons call their departmental colleagues and nurses to help in CPR.

Conclusions: An insufficient formal education in CPR was registered for all groups, reflecting the lack of familiarity with new CPR methods. A team education, involving doctors and nurses may improve familiarity with CPR and patient outcomes.
\end{abstract}

Keywords: Cardiopulmonary Resuscitation; Education; Guideline Adherence; Health Knowledge, Attitudes, Practice; Infection

\section{Background}

In the field of cardiopulmonary resuscitation (CPR) numerous improvements including electric defibrillation and closed chest cardiac massage were introduced but the survival of victims of cardiac arrest is still poor (1). European Resuscitation Council (ERC) has released new guidelines in 2010 based on the results of systematic reviews and clinical trials (2).

The importance of education was highlighted in the new ERC guidelines (2). It aimed at both acquisition and retention of technical skills i.e. early recognition of cardiopulmonary arrest, performance of CPR, and nontechnical skills such as organization and leadership (3). These basic resuscitation skills deteriorate within three to six months, and need renewal $(4,5)$. Although research about the impact of continuous education on patients' outcome is missing, it is likely that performance of CPR may be significantly improved after training $(5,6)$.

Chest compression-only CPR is a recently introduced method of basic life support in non-asphyxial arrest and during the first few minutes after cardiac arrest it may increase survival. This method is, therefore, recommended by the ERC as a method of choice for CPR delivered by lay people and untrained rescuers who are unable or unwilling to give rescue breaths (2). The ERC encourages this method for telephone-guided resuscitation, for rescuers having insufficient knowledge on the traditional CPR until awaiting professional help (2).

Chest compression-only is not as effective as conventional resuscitation, but is preferred over no resuscita-

Copyright ( 2015, Iranian Red Crescent Medical Journal. This is an open-access article distributed under the terms of the Creative Commons Attribution-NonCommercial 4.0 International License (http://creativecommons.org/licenses/by-nc/4.0/) which permits copy and redistribute the material just in noncommercial usages, provided the original work is properly cited. 
tion. Chest-compression-only resuscitation may also be preferred by medical professionals when there is a significant risk of disease transmission and no barrier devices are present. It is a sufficient resuscitation method for the first few minutes after cardiac arrest, since arterial oxygen stores become depleted after two to four minutes (2). This method is not recognized by medical professionals since it is still somewhat new.

Disease transmission is only occasionally reported after CPR $(7,8)$. In a meta-analysis from year 1998, Mejicano and Maki found that only 15 documented cases, mainly bacterial infections, were reported after mouthto-mouth ventilation. Another three cases of HIV infection during CPR resulted from high-risk cutaneous exposures (9). Both fear from infection and insufficient medical knowledge may result in the avoidance of resuscitation (10, 11). Attitudes towards cardiopulmonary resuscitation are probably different in subsets of health professionals (12). These may be particularly different between doctors, who are highly specialized in performing specific surgical procedure diagnostics, and anesthesiologists, who perform resuscitations more often.

\section{Objectives}

In this study we attempted to compare education in CPR, current call-for-help practices, team-work and attitudes about key points of the resuscitation process in a group of anesthesiologists, who are assumed to be skilled in resuscitation, with surgeons and medical doctors. Chest-compression-only resuscitation, anticipated risk of infection related to mouth-to-mouth ventilation and discontinuation of CPR may be indicators of their continuous education.

\section{Patients and Methods}

After institutional approval (No. 021-02/12) was obtained, a survey was conducted among doctors, specialists and residents at the Osijek University Hospital. This hospital is a tertiary 1200-bed community teaching hospital. During the period from 8th to 12th of April 2012 a total of 228 doctors were eligible for participation in this cross sectional study and were given anonymous questionnaires by a single observer (supplementary file 1). A total of 195 doctors returned valid questionnaires. Five questionnaires were not correctly completed and were excluded, and finally 190 questionnaires were analyzed. The study population consisted of 111 (58.4\%) male and 79 (41.6\%) female doctors. For the study purposes, 51 specialists in internal medicine, 9 radiologists, 12 neurologists, 4 specialists for infective diseases, and 17 pediatricians were assigned to the medical group ( $\mathrm{n}$ =93). A subset of 27 general surgeons, 14 gynecologists, 19 otorhinolaryngologists and maxillofacial surgeons, and 10 specialists in neurosurgery were assigned to the surgical group $(\mathrm{n}=70)$. Their answers were compared with 27 anesthesiologists.

\subsection{Statistical Analysis}

Data were analyzed using the SPSS 18.0 software for Windows. Demographic data were shown as means and standard deviations and analyzed using the ANOVA test. Fisher's exact probability test, odds ratio and 95\% confidence intervals were calculated for categorical data. A relationship between variables was calculated using Spearman's correlation coefficient $r$. P value $<0.05$ was considered statistically significant.

\section{Results}

Demographic data of respondents are presented in Table 1 . No differences were observed regarding mean age, years in practice, and residents and specialists' ratio between groups. More male doctors were in the group of surgical specialists as compared to the anesthesiologists (OR 5.33; 95\% CI $=2.05$ to $13.88, \mathrm{P}<0.001$ ) and medical doctors (OR 3.91; 95\% CI $=1.94$ to $7.88, \mathrm{P}<0.001$ ).

Medical doctors reported significantly more resuscitations on their departments as compared to surgeons, whereas almost all anesthesiologists reported $>20$ resuscitations per year $(\mathrm{p}=0.003$, Figure 1$)$. The greatest number of resuscitations was reported by subgroup of anesthesiologists, neurologists and general surgeons, who mainly reported more than 20 resuscitations per year. A great proportion of doctors in these three subgroups reported that they haven't systematically renewed their knowledge in resuscitation within the last ten years ( 6 in 12 neurologists, and 13 in 28 general surgeons compared to 6 in 27 (anesthesiologists, $\mathrm{P}=0.108$ ). Departmental staff initiates resuscitations in all departments (Table 2). Regarding the person starting resuscitation, organization was similar between the three groups. More than half of doctors in all groups, i.e. 60 out of 93 in medical, 35 of 70 in surgical group and 19 of 27 anesthesiologists, responded that either the doctor or nurse start resuscitation at their departments ( $P$ $=0.413$ ). Our respondents mentioned that nurses alone only rarely started resuscitation in all groups (six in medical, five in surgical, and one in anesthesia group).

Similar answers were given regarding call for help. More than half of the respondents in all groups reported that both doctors and nurses call for help (57\% in medical, 50\% in surgical and 52\% in the group of anesthesiologists; $\mathrm{P}=$ 0.564 ). One in three medical doctors (36 in 93), and half of anesthesiologists call their departmental colleagues for help in resuscitation, whereas only seven in 70 surgeons would call another surgeon $(\mathrm{OR}=5.68,95 \%$ CI 2.34- 13.77; $\mathrm{P}<0.001$ ). Almost all surgeons (69 in 70) would call the attending anesthesiologist for help compared with 79 in 93 medical doctors (OR 0.08; 95\% CI $0.01-0.63$; $\mathrm{P}=0.004$ ). Only seven surgeons and five anesthesiologists would call departmental nurses for help. In contrast 22 medical doctors will call nurses for help $(\mathrm{P}=0.024)$.

Most of the respondents reported that they are personally involved in resuscitation at their department 
(Table 2). In the group with 14 doctors who did not participate in resuscitations at their departments there were 10 female doctors and only four male doctors (OR $3.78,95 \% \mathrm{CI}=0.14-12.5 ; \mathrm{P}=0.018)$. The majority of anesthesiologists and medical doctors responded that they start with reanimation each time when necessary, yet 14 in 70 surgeons responded that they never had an opportunity to resuscitate any patient. Medical doctors start resuscitation alone more readily than surgeons, yet this difference was not significant (90.3\% vs. 80\%; P $=0.060$ ).

Attitudes of doctors regarding resuscitation and infections were not significantly different. In case of out of hospital resuscitation, $40 \%$ of medical doctors and surgeons and 17 (62\%) of anesthetists considered infection risk as significant during mouth-to-mouth ventilation. Anesthesiologists were more likely to refuse rescue breaths due to the possibility of infection as compared to others, yet these differences were not significant $(\mathrm{P}=0.159$, Table 2$)$.

When asked about the risk of infection during mouthto-mouth ventilation, nine anesthesiologists, 10 surgeons and 20 medical doctors considered the risk of infection real and higher than $10 \%$. On average anesthesiologists thought that the risk of infection is higher as compared to the other two groups. Differences were statistically significant between anesthesiologists and surgeons $(\mathrm{P}=0.032)$, whereas medical doctors did not differ significantly from their colleagues $(\mathrm{P}=0.912)$. A correlation analysis revealed that doctors who were afraid of infection estimated a high infection risk. These doctors would reject mouth-to-mouth resuscitation more readily, and claimed that chest-compressiononly resuscitation is acceptable $(r=0.275$; $\mathrm{P}<0.001)$. Anesthesiologists, in contrast to medical doctors and surgeons, thought that chest-compression-only may be an acceptable resuscitation method when the rescuer is unwilling to give rescue breaths $(\mathrm{P}<0.001)$

Even though doctors predominantly considered their knowledge on CPR important (Table 2), this knowledge was not supported by their education on CPR. Approximately $45 \%$ of medical doctors, $48 \%$ of surgeons and $77 \%$ of anesthesiologists reported that they had renewed their knowledge on CPR within the last five years $(\mathrm{P}<0.001)$. One third $(34 \%)$ of medical doctors and $25 \%$ of surgical specialists reported that they had never renewed their knowledge on CPR after they had completed their medical study. A greater number of female respondents had never undergone education in resuscitation (36\% females vs. $28 \%$ males, $\mathrm{P}=0.339$ ). Differences regarding education were not observed between specific age subsets. When asked about how often resuscitation guidelines are being changed, 24 (88\%) anesthesiologists, 40 (57\%) surgeons, and only 26 (28.2\%) medical doctors gave correct answers $(\mathrm{P}<0.001$ between anesthesiologists and medical doctors or surgeons).

All anesthesiologists were familiar with resuscitation equipment, and reported an average of 3.2 resuscitation tools, or stated that they had all the available tools, whereas 13 medical doctors and 20 surgeons answered that they did not know which equipment they had. Although medical doctors on average mentioned more resuscitation tools than surgeons (2.1 vs. 1.2), this difference was not statistically significant. A total of 88 doctors mentioned that they had a self-inflating bag, 60 listed defibrillators, and 35 laryngoscopes. Interestingly, five doctors specified an anesthesia machine, and one central venous catheter and central venous pressure monitoring as resuscitation equipment.

No difference was observed regarding doctors' personal opinion on the cessation of resuscitation. More than half of medical doctors, surgeons and anesthesiologists (45, 33 and 16) considered that resuscitation of adult patients may be stopped after 30 minutes, whereas 18,19 , and 3 doctors in those groups considered that it may be stopped after 20 minutes $(P>0.3)$.

Table 1. Demographic Data of Respondents

\begin{tabular}{|c|c|c|c|c|}
\hline Variables & $\begin{array}{l}\text { Medical Doctors } \\
\qquad(\mathbf{N}=93)\end{array}$ & $\begin{array}{l}\text { Surgeons } \\
(N=70)\end{array}$ & $\begin{array}{l}\text { Anesthesiologists } \\
\qquad(\mathbf{N}=\mathbf{2 7})\end{array}$ & PValues \\
\hline Mean age, $\mathrm{y}^{\mathrm{a}}$ & $42.7 \pm 10.4$ & $40.1 \pm 9.6$ & $43.7 \pm 10.5$ & 0.190 \\
\hline Years in practice $^{a}$ & $16.6 \pm 10.5$ & $13.55 \pm 9.6$ & $16.7 \pm 10.7$ & 0.146 \\
\hline Gender, $\mathbf{n}$ & & & & $<0.001^{b}$ \\
\hline Male & 45 & 55 & 11 & \\
\hline Female & 48 & 15 & 16 & \\
\hline Residents: specialists & $23: 70$ & $19: 51$ & $5: 22$ & 0.718 \\
\hline Professor/assistant at the Medical Faculty, \% & 46 & 34 & 30 & 0.234 \\
\hline
\end{tabular}

${ }^{\mathrm{a}}$ Values are presented as mean $\pm \mathrm{SD}$.

b Statistically significant difference was determined using the Fisher Exact Probability Test. 
Krajina I et al.

Table 2. Resuscitation Practices at the Osijek University Clinical Hospital a, b, c

\begin{tabular}{|c|c|c|c|c|}
\hline Resuscitation Practices & $\begin{array}{l}\text { Medical Doctors } \\
\quad(\mathbf{n}=93)\end{array}$ & $\begin{array}{l}\text { Surgeons } \\
(\mathbf{n}=70)\end{array}$ & $\begin{array}{l}\text { Anesthesiologist } \\
\quad(\mathbf{n}=\mathbf{2 7})\end{array}$ & PValues \\
\hline Do your department staffs start CPR alone? & $88(94.6)$ & $68(97.1)$ & $27(100)$ & 0.658 \\
\hline Are you personally involved in resuscitation at your department? & $82(88.1)$ & $65(93)$ & $27(100)$ & 0.147 \\
\hline $\begin{array}{l}\text { Do you think that knowledge in resuscitation is important for } \\
\text { your profession? }\end{array}$ & $91(97.8)$ & $64(91)$ & $27(100)$ & 0.086 \\
\hline $\begin{array}{l}\text { If you should give rescue breath in public places (i.e. bus station) } \\
\text { would you be afraid of infection? }\end{array}$ & $43(46)$ & $31(44.3)$ & $17(63)$ & 0.239 \\
\hline $\begin{array}{l}\text { Would you deny rescue breaths when called for help because of } \\
\text { risk of infection? }\end{array}$ & $14(15.1)$ & $7(10)$ & $7(25.9)$ & 0.159 \\
\hline $\begin{array}{l}\text { Do you think that chest-compression-only resuscitation may be ac- } \\
\text { ceptable when the doctor is unwilling to give rescue breaths? }\end{array}$ & $24(25.8)$ & $10(14.3)$ & $16(59.3)$ & 0.001 \\
\hline
\end{tabular}

a Values are presented as No. (\%).

b Number of respondents in each group who gave positive answers is shown and the ratio within the group in parentheses.

c Differences were calculated using the Fisher exact probability test between medical doctors and surgeons, between medical doctors and anesthesiologists, and between surgeons and anesthesiologists.

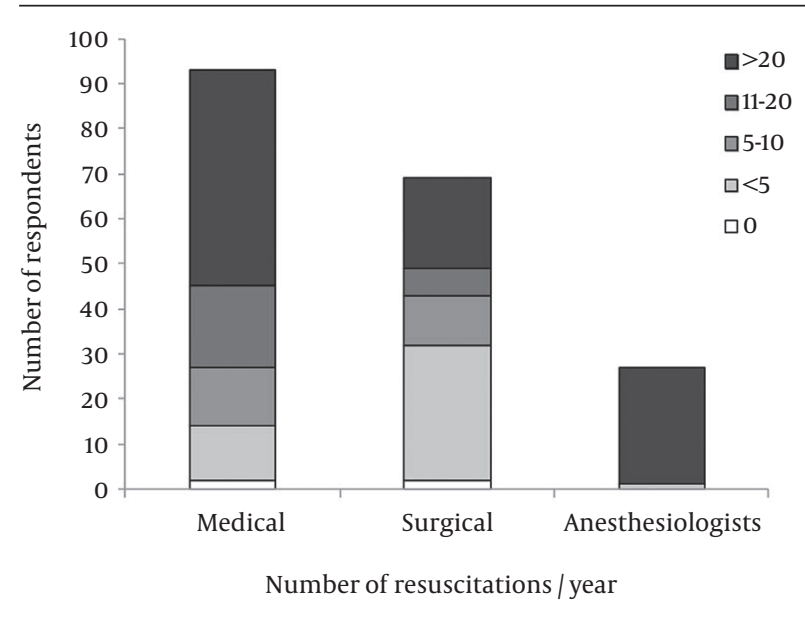

Figure 1. Number of resuscitations in their department/emergency care unit reported by each respondent

\section{Discussion}

This study confirmed that the three groups of respondents had different education and attitudes towards resuscitations. The group of anesthesiologists was more aware about new guidelines and chest-compression-only resuscitation as a new resuscitation method, and they had renewed their knowledge more recently when compared with other groups. Their attitudes regarding resuscitation were different compared with medical doctors and surgeons.

A call for help is a significant step in the chain of survival as proposed by the European Resuscitation Council (2). In the hospital environment this may reflect both local practices and trust towards colleagues' competence. In our study group, different attitudes were observed between medical and surgical specialists. Medical doctors, who perform more resuscitation, readily call their departmental colleagues and nurses for help during the resuscitation process, a practice which was not observed in the surgical group. A change in the practices of in-hospital resuscitation, with recognition of critical illness, staff education, early call for help, and team-work may prevent a significant number of in-hospital cardiac arrests. By adopting this view, "chain of survival" may arise to "chain of prevention" $(2,13)$.

Most doctors in the three groups claimed that knowledge in resuscitation is important for them and that they are members of resuscitation teams. The performance of resuscitation depends on both technical skills such as ventilation and chest compression, and nontechnical skills such as leadership and teamwork $(3,4,6,11,14)$. This procedure may be particularly important for departments with higher incidence of cardiorespiratory arrests. Despite this, our study did not confirm a correlation between education and number of resuscitations at particular departments. This situation may arise from the fact that knowledge and performance of CPR may not be of prime importance for neurologists and surgeons, who equivocally call anesthesiologists for help at our institution.

Based on the results of this survey, only a minority of doctors would call nurses to help during resuscitation. For this specific issue, departmental nurses are not considered as partners. Our observation may be a result of adopted beliefs that nurses are probably not as equally effective as doctors $(15,16)$. This common opinion emerged from insufficient nurses' education and their inadequate knowledge in the CPR $(15,16)$.

Assuming that doctors are focused on specific professional interests, while nurses are more dedicated to patient care, it is obvious that they may first recognize cardiac arrest and start resuscitation before doctors. This fact may be an important point for improvements, because nurses spend more of their work hours directly with patients. Nurses alone start resuscitations usually after consultation with the doctor and this process may 
take a few critical minutes. Nurses' education in principal resuscitation techniques may be of prime importance $(14,17)$. An early recognition of cardiac arrest and early uninterrupted bystander CPR is pointed in the new ERC recommendations (2). A method to improve these steps may be team-work education of both nurses and doctors. As retention of resuscitation skills deteriorates over time, such education should be periodically repeated $(14,18)$, and should not be left to personal initiatives.

A few recent studies have confirmed that nurses are equally as effective in CPR as doctors, if they have appropriate education in CPR (19). Until now there are no studies investigating whether patient survival may be improved by upgrading nurses from passive assistants to clinically competent resuscitation providers.

A familiarity with equipment is one of the indicators in the assessment of knowledge and skills (20). A notable proportion of surgeons and medical doctors weren't able to list resuscitation equipment at their department. This common problem may impede care of cardiac arrest victims, but may be overcome by training (21). All residents may become a target group for education in airway management and CPR (21). An implementation of rotations in anesthesiology as an obligation during education may improve familiarity of residents with equipment and performance of CPR.

In this survey a relatively high infection risk was estimated for rescuers, during mouth-to-mouth ventilation by all respondents. Anesthesiologists estimated disproportionately high infection risk and refused mouth-tomouth ventilation when being called for help in public places. On the contrary, surgeons who are rarely performing resuscitations estimated a lower risk. Their willingness to perform CPR, is supported neither by familiarity with resuscitation tools, nor with education in CPR and recognition of recommended resuscitation methods.

A relatively high reluctance of healthcare providers to perform mouth-to mouth ventilation in a public general hospital was reported by Giammaria and coworkers in their study during year 2005 (22). They found that as much as $58 \%$ of healthcare providers would not perform mouth-to-mouth ventilation without barrier devices; and 90.6\% would perform BLS only by chest compression (22). Recent guidelines and literature reports have suggested that early-uninterrupted chest-compression-only resuscitation enhances the probability of survival in cardiac arrest victims (2). Since chest-compression-only resuscitation is a new technique, it was not been accepted by our three study groups, with only $25 \%$ of doctors supporting its utility. Anesthesiologists were the most familiar with this method. Since anesthesiologists are commonly called to help with resuscitations in our institution, this method of resuscitation must be recommended to untrained departmental stuff until the qualified resuscitation team arrives. With implementing basic education and teamwork in CPR, knowledge and performance of resuscitation may be improved among all doctors and nurses.
The weak point of this study is that it did not investigate knowledge in specific CPR procedures. Such evaluation should give more data, and aid in the development of CPR education. It may be performed on manikins before and after CPR courses, rather than using questionnaires (14). After repetitive CPR training with assessment of psychomotor skills, the performance of CPR may be retained and new procedures implemented. In that way prevention of cardiac arrests and early treatment of pre-arrest conditions may save lives in the hospital environment, as pointed in the ERC guidelines (2).

In conclusion, different attitudes and knowledge with lack of systemic education and team collaboration were found in our three study groups. The risk of infection transmission during resuscitation was overestimated by all groups, while chest-compression-only was not recognized as a valuable method of resuscitation. Team education of both doctors and nurses, and implementation of obligatory CPR courses as a method of continuous education may improve the understanding and performance of resuscitation, and consequently patients' outcome.

\section{Authors' Contributions}

Irena Krajina, Slavica Kvolik and Kristina Kovacevic were responsible for the study concept and design. Acquisition of data was done by Irena Krajina. Robert Steiner, Irena Krajina and Slavica Kvolik were responsible for analysis and interpretation of data. Slavica Kvolik, Irena Krajina and Ivan Lovric drafted the manuscript. Slavica Kvolik, Irena Krajina and Robert Steiner performed the critical revision of the manuscript for important intellectual content.

\section{References}

1. Ruzman T, Tot OK, Ivic D, Gulam D, Ruzman N, Burazin J. In-hospital cardiac arrest: can we change something? Wien Klin Wochenschr. 2013;125(17-18):516-23.

2. Nolan JP, Soar J, Zideman DA, Biarent D, Bossaert LL, Deakin C, et al. European Resuscitation Council Guidelines for Resuscitation 2010 Section 1. Executive summary. Resuscitation. 2010;81(10):1219-76.

3. Hayes CW, Rhee A, Detsky ME, Leblanc VR, Wax RS. Residents feel unprepared and unsupervised as leaders of cardiac arrest teams in teaching hospitals: a survey of internal medicine residents. Crit Care Med. 2007;35(7):1668-72.

4. Grzeskowiak M, Bartkowska-Sniatkowska A, Rosada-Kurasinska J. A survey of anaesthesiology residents' knowledge of resuscitation guidelines. Anestezjol Intens Ter. 2010;42(4):187-9.

5. Koster RW, Baubin MA, Bossaert LL, Caballero A, Cassan P, Castren M, et al. European Resuscitation Council Guidelines for Resuscitation 2010 Section 2. Adult basic life support and use of automated external defibrillators. Resuscitation. 2010;81(10):1277-92.

6. Kallestedt ML, Berglund A, Herlitz J, Leppert J, Enlund M. The impact of CPR and AED training on healthcare professionals' selfperceived attitudes to performing resuscitation. Scand J Trauma Resusc Emerg Med. 2012;20:26.

7. Arend CF. Transmission of infectious diseases through mouth-tomouth ventilation: evidence-based or emotion-based medicine? Arq Bras Cardiol. 200 0;74(1):86-97.

8. Wenzel V, Idris AH, Dorges V, Nolan JP, Parr MJ, Gabrielli A, et al. The respiratory system during resuscitation: a review of the history, risk of infection during assisted ventilation, respiratory 
mechanics, and ventilation strategies for patients with an unprotected airway. Resuscitation. 2001;49(2):123-34.

9. Mejicano GC, Maki DG. Infections acquired during cardiopulmonary resuscitation: estimating the risk and defining strategies for prevention. Ann Intern Med.1998;129(10):813-28.

10. Horowitz BZ, Matheny L. Health care professionals' willingness to do mouth-to-mouth resuscitation. West J Med.1997;167(6):392-7.

11. Brenner B, Stark B, Kauffman J. The reluctance of house staff to perform mouth-to-mouth resuscitation in the inpatient setting: what are the considerations? Resuscitation. 1994;28(3):185-93.

12. Brenner BE, Van DC, Lazar EJ, Camargo CA,Jr. Determinants of physician reluctance to perform mouth-to-mouth resuscitation. J Clin Epidemiol. 2000;53(10):1054-61.

13. Smith GB. In-hospital cardiac arrest: is it time for an in-hospital 'chain of prevention'? Resuscitation. 2010;81(9):1209-11.

14. Mokhtari Nori J, Saghafinia M, Kalantar Motamedi MH, Khademol Hosseini SM. CPR Training for Nurses: How often Is It Necessary? Iran Red Crescent Med J. 2012;14(2):104-7.

15. Kallestedt ML, Rosenblad A, Leppert J, Herlitz J, Enlund M. Hospital employees' theoretical knowledge on what to do in an in-hospital cardiac arrest. Scand J Trauma Resusc Emerg Med. 2010;18:43.

16. Preusch MR, Bea F, Roggenbach J, Katus HA, Junger J, Nikendei C. Resuscitation Guidelines 2005: does experienced nurs- ing staff need training and how effective is it? Am J Emerg Med. 2010;28(4):477-84.

17. Hunziker S, Johansson AC, Tschan F, Semmer NK, Rock L, Howell $\mathrm{MD}$, et al. Teamwork and leadership in cardiopulmonary resuscitation. J Am Coll Cardiol. 2011;57(24):2381-8

18. Morgan R, Westmoreland C. Survey of junior hospital doctors' attitudes to cardiopulmonary resuscitation. Postgrad Med J. 2002;78(921):413-5.

19. Gilligan P, Bhatarcharjee C, Knight G, Smith M, Hegarty D, Shenton A, et al. To lead or not to lead? Prospective controlled study of emergency nurses' provision of advanced life support team leadership. Emerg Med J. 2005;22(9):628-32.

20. Hunziker S, Tschan F, Semmer NK, Howell MD, Marsch S. Human factors in resuscitation: Lessons learned from simulator studies. J Emerg Trauma Shock. 2010;3(4):389-94.

21. Eppich WJ, Zonfrillo MR, Nelson KL, Hunt EA. Residents mental model of bag-mask ventilation. Pediatr Emerg Care. 2010;26(9):646-52.

22. Giammaria M, Frittelli W, Belli R, Chinaglia A, De Michelis B, Ierna $S$, et al. [Does reluctance to perform mouth-to-mouth ventilation exist among emergency healthcare providers as first responders?]. Ital Heart J Suppl. 2005;6(2):90-104. 